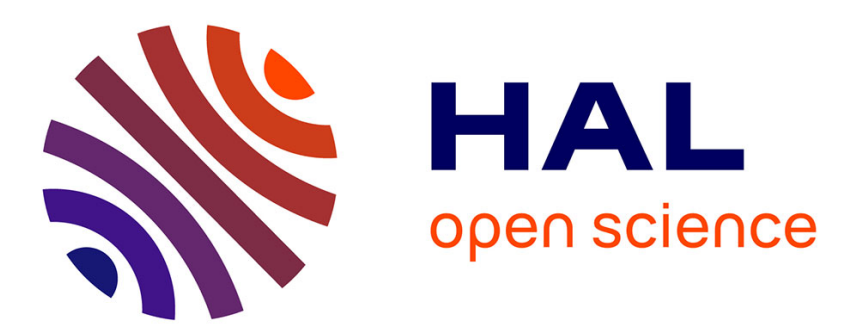

\title{
LMI-Based invariant like nonlinear state observer for anaerobic digestion model
}

\author{
Khadidja Chaib Draa, Marouane Alma, Holger Voos, Ali Zemouche,
} Mohamed Darouach

\section{- To cite this version:}

Khadidja Chaib Draa, Marouane Alma, Holger Voos, Ali Zemouche, Mohamed Darouach. LMI-Based invariant like nonlinear state observer for anaerobic digestion model. 25th Mediterranean Conference on Control and Automation, MED 2017, Jul 2017, Valletta, Malta. 10.1109/med.2017.7984197 . hal-01567358

\section{HAL Id: hal-01567358 \\ https://hal.science/hal-01567358}

Submitted on 12 Jan 2018

HAL is a multi-disciplinary open access archive for the deposit and dissemination of scientific research documents, whether they are published or not. The documents may come from teaching and research institutions in France or abroad, or from public or private research centers.
L'archive ouverte pluridisciplinaire HAL, est destinée au dépôt et à la diffusion de documents scientifiques de niveau recherche, publiés ou non, émanant des établissements d'enseignement et de recherche français ou étrangers, des laboratoires publics ou privés. 


\title{
LMI-Based Invariant Like Nonlinear State Observer for Anaerobic Digestion Model
}

\author{
K. Chaib Draa, M. Alma, H. Voos, A. Zemouche and M. Darouach
}

\begin{abstract}
This note deals with the design of an invariant like nonlinear state observer for a two step (acidogenesismethanogenesis) mass balance nonlinear model. In order to ensure the stability of the estimation error, a new LMI condition is proposed. The feasibility of this LMI is enhanced due to the use of a suitable reformulation of the Youngs inequality. Actually, additional decision variables are included in the LMI to render its feasibility less conservative compared to those established in the literature for the same class of systems. The numerical simulations using the investigated anaerobic digestion model show the effectiveness of the proposed LMI methodology.
\end{abstract}

Index Terms-Anaerobic digestion, LMI approach, Observer Design, Nonlinear systems.

\section{INTRODUCTION}

Anaerobic digestion (AD) is one of the most optimal ways to convert organic waste into useful energy [23], [15]. The process occurs inside an anaerobic digester where the organic matter is transformed into biogas through several biologic reactions, including different species of micro-organisms. Often, the produced biogas is converted into electrical energy and injected to the power grid.

Usually, in biological processes things seem to work fairly well and reasobably until some failures or faults occur [12]. This may be due to the specific behaviour of the system itself or to the presence of disturbances which can highly affect it. Thus, an obvious need for an efficient control and monitoring of such systems arises.

However, a basic difficulty facing the process monitoring is a shortage in biosensors which makes the process state very difficult to characterize [2]. To overcome this issue, a couple of software sensors have been proposed in the literature. Among them, we can cite the asymptotic observer reported in [3] which is quite simple and does not require the knowledge of some specific non linear functions. However, it has a drawback concerning the speed of convergence which is equal to the control input. Therefore, a more appropriate solution has been proposed in [10], [19] when the time-varying bounds enclosing the uncertainties are known. Indeed, the solution in this case is the use of interval observer

Khadidja CHAIB DRAA is with the Interdisciplinary Centre for Security, Reliability and Trust (SnT)- Université du Luxembourg and with Université de Lorraine, CRAN CNRS UMR 7039, 54400 Cosnes et Romain, France. khadidja.chaibdraa@uni.lu

Holger VOOS is with the Interdisciplinary Centre for Security, Reliability and Trust (SnT)- Université du Luxembourg.

Marouane ALMA and Mohamed DAROUACH are with the CRAN-CNRS, Université de Lorraine, IUT de Longwy.

Ali ZEMOUCHE is with Université de Lorraine, CRAN CNRS UMR 7039, 54400 Cosnes et Romain, France, and with EPI INRIA DISCO, Laboratoire des Signaux et Systmes, CNRS-Supélec, 91192 Gif-sur-Yvette, France. which is composed, usually, of two asymptotic observers, one to estimate the state upper bound $x^{+}(t)$ and a second one to estimate the lower bound $x^{-}(t)$, and then the actual state will be enclosed in the envelope drown by $x^{-}(t)$ and $x^{+}(t)$ provided that at the initial time $x^{-}\left(t_{0}\right) \leq x\left(t_{0}\right) \leq x^{+}\left(t_{0}\right)$. Unfortunately, it happens sometimes to obtain a large envelope of the state and thus the estimation becomes useless. Therefore, Bernard and his co-authers [4] have proposed to launch a bundle of interval observers with different initial conditions and then select the best (smallest) envelope. The advantage of the bundle observers is the improvement of the asymptotic bounds and the ability to partially tune the rate of convergence. This is possible due to the use of additional measurements which are non linear functions of the state variables. However, the interval observers are applied only when the dynamic of the estimation error is cooperative ${ }^{1}$. Therefore, the interval observers can be applied only for a specific class of systems. In addition to that, it is not easy to exploit the estimated intervals for control.

For reliable models, the extended Kalman filter (based on a linear approximation of the non linear process model) has been extensively applied to the AD applications [3], [16], [6], [9], [8] and the references in [13], with success and has even covered the situations where measurements of the process outputs arrive at different sampling rates [21]. However, unfortunately it has been prooved in [17] that due to the non linear structure of the AD models and the number of input variables, it becomes impossible to apply the extended Kalman filter when one wants to estimate the biomass concentration (the system is not uniformly observable for any input), which is a key state variable to estimate since it reflects the system health and stability [18].

A new kind of observers has been proposed in [1], [22] and applied to a class of chemical reactors, they are called invariant observers, where the invariance refers to the invariance under a group action, and are based on Lie group symmetries. The advantage of these observers is their adjustable rate of convergence as it has been shown in [5]. Moreover, the invariant observer has been applied successfully in [7] to a forth order AD model with the use of only cheap and commonly done measurement. Thus, inspired by the structure of the invariant observer and motivated by the estimation of bacteria concentrations in AD models, we design in the current work a novel LMI based invariant like nonlinear observer for a sixth order acidogenesis-methanogenesis mass balance nonlinear model.

${ }^{1}$ All elements of the Jacobian matrix of the system are positive. 
The rest of the paper is organised as follows. In Section II, we present the studied model, then in Section III, we detail the designed observer and give the proof of convergence of the estimation error. In Section IV, we run numerical simulation to illustrate the theoretical findings. Finally, in Section V, we conclude the paper.

\section{Model of Anaerobic Digestion Process}

The AD process is represented by the nonlinear mathematical model proposed in [20], [11] and slightly modified in [14], where the acidogenesis and methanogenesis reactions are considered to be the limiting steps, and are modelled by the following equations:

$$
\begin{aligned}
\dot{x}_{1}= & -k_{1} \mu_{1}\left(x_{1}\right) x_{2}+u_{1} S_{1 \text { in }}-u_{\text {out }} x_{1} \\
\dot{x}_{2}= & \mu_{1}\left(x_{1}\right) x_{2}-u_{\text {out }} x_{2} \\
\dot{x}_{3}= & k_{2} \mu_{1}\left(x_{1}\right) x_{2}-k_{3} \mu_{2}\left(x_{3}\right) x_{4}-u_{\text {out }} x_{3}+ \\
& u_{1}\left(S_{2 \text { in }}+S_{2 a d}\right) \\
\dot{x}_{4}= & \mu_{2}\left(x_{3}\right) x_{4}-u_{\text {out }} x_{4} \\
\dot{x}_{5}= & k_{4} \mu_{1}\left(x_{1}\right) x_{2}+k_{5} \mu_{2}\left(x_{3}\right) x_{4}+u_{1} C_{\text {in }}- \\
& u_{\text {out }} x_{5}-Q_{c}(x) \\
\dot{x}_{6}= & u_{1} Z_{\text {in }}+u_{2} Z_{\text {ad }}-u_{\text {out }} x_{6}
\end{aligned}
$$

where $x_{1}(g / l)$ represents concentration of the organic matter to be digested, and $x_{2}(g / l)$ concentration of acidogenic bacteria which degrades $x_{1}$. The volatile fatty acids concentration $x_{3}(\mathrm{mmol} / \mathrm{l})$ is supposed to be pure acetate, $x_{4}(g / l)$ is concentration of methanogenic bacteria, $x_{5}(\mathrm{mmol} / \mathrm{l}) \mathrm{rep}-$ resents the inorganic carbon concentration and $x_{6}(\mathrm{mmol} / \mathrm{l})$ the alkalinity concentration. The control inputs are $u_{1}=\frac{F_{1 i n}}{v}$ $\left(\frac{1}{\text { day }}\right)$ and $u_{2}=\frac{F_{2 i n}}{v}\left(\frac{1}{\text { day }}\right)$, where $F_{1 \text { in }}$ is the input flow rate of waste to the digester and $F_{2 \text { in }}$ input flow rate of the added alkalinity $\left(Z_{a d}\right)$. Since the digester volume $(v)$ is constant the output dilution rate $u_{\text {out }}=u_{1}+u_{2}$. Regarding the gas flow rates, we have $Q_{m}(x)$ and $Q_{c}(x)$ which represent the methane and $\mathrm{Co}_{2}$ gas flow rates, respectively. We also have the following equations, which express the relationships between bicarbonates ( $\mathrm{bic}$ ), dissolved $\mathrm{Co}_{2}$ and the $\mathrm{pH}$ :

$$
\left\{\begin{array}{l}
\text { bic }=x_{6}-x_{3} \\
c o_{2}=x_{5}-b i c \\
k_{b}=\frac{\left[H^{+}\right] b i c}{c o_{2}} \\
p H=-\log _{10}\left(k_{b} \frac{c o_{2}}{b i c}\right)
\end{array}\right.
$$

where $k_{b}$ is the acidity constant of bicarbonates (bic).

The bacteria growth rate functions are of type Monod for $x_{2}$ and of type Haldane for $x_{4}$ :

$$
\left\{\begin{array}{l}
\mu_{1}\left(x_{1}\right)=\bar{\mu}_{1} \frac{x_{1}}{x_{1}+k_{s_{1}}} \\
\mu_{2}\left(x_{3}\right)=\bar{\mu}_{2} \frac{x_{3}}{x_{3}+k_{s 3}+\frac{x_{3}^{2}}{k_{i 3}}}
\end{array}\right.
$$

Gaseous flow rates of methane $\left(Q_{m}(x)\right)$ and carbon dioxide
$\left(Q_{c}(x)\right)$ are expressed as the following

$$
\left\{\begin{array}{l}
Q_{m}(x)=k_{6} \mu_{2}\left(x_{3}\right) x_{4} \\
c o_{2}=x_{5}+x_{3}-x_{6} \\
Q_{c}(x)=\frac{R T \gamma_{c o}}{P_{T}+R T \gamma\left(K_{H} P_{T}-c_{2}\right)} Q_{m}(x)
\end{array}\right.
$$

The concentrations $S_{1 i n}, S_{2 i n}, C_{i n}$ and $Z_{i n}$ correspond to the fed waste characteristics and the concentration $S_{2 a d}$ is an additional input which represents the added acids to the fed waste.

The system output is divided into linear $\left(y_{1}\right)$ and nonlinear $\left(y_{2}\right)$ outputs

$$
\left\{\begin{array}{l}
y_{1}=\left[x_{1}, x_{3}, x_{6}\right]^{T} \\
y_{2}=Q_{c}(x)
\end{array}\right.
$$

All parameters used in the previous equations and not defined up to now are determined in Table I.

\section{OBSERVER DESIGN}

In this section, we present the designed nonlinear invariant like observer that we use to estimate the states which are the most costly and difficult to measure in the AD process, bacteria concentrations, and inorganic carbon concentration. Fortunately the proposed observer can be designed for different class of systems. Consequently, we present the results in a general way.

In order to refine the presentation of the findings, we first provide some useful preliminaries.

\section{A. Some useful preliminaries}

The following preliminaries are very useful to ensure the asymptotic convergence of the state observer that we will propose later.

Theorem 1 (Mean value theorem [24]): Let $\varphi: \mathbb{R}^{n} \rightarrow$ $\mathbb{R}^{q}$. Let $x, y \in \mathbb{R}^{n}$. We assume that $\varphi$ is differentiable on $\operatorname{Co}(x, y)$. Then, there are constant vectors $z_{1}, \ldots, z_{q} \in$ $\operatorname{Co}(x, y), z_{i} \neq x, z_{i} \neq y$ for $i=1, \ldots, q$ such that :

$$
\varphi(x)-\varphi(y)=\left(\sum_{i, j=1}^{q, n} e_{q}(i) e_{n}^{T}(j) \frac{\partial \varphi_{i}}{\partial x_{j}}\left(z_{i}\right)\right)(x-y) .
$$

Lemma 3.1 (a variant of Lipschitz reformulation): Let $\varphi: \mathbb{R}^{n} \rightarrow \mathbb{R}^{q}$ a differentiable function on $\mathbb{R}^{n}$. Then, the following items are equivalent:

- $\varphi$ is a globally $\gamma_{\varphi}$-Lipschitz function;

- there exist finite and positive scalar constants $a_{i j}, b_{i j}$ so that for all $x, y \in \mathbb{R}^{n}$ there exist $z_{i} \in \operatorname{Co}(x, y), z_{i} \neq x, z_{i} \neq y$ and functions $\psi_{i j}: \mathbb{R}^{n} \rightarrow \mathbb{R}$ satisfying the following:

$$
\begin{gathered}
\varphi(x)-\varphi(y)=\sum_{i, j=1}^{i, j=q, n} \psi_{i j}\left(z_{i}\right) \mathscr{H}_{i j}(x-y) \\
a_{i j} \leq \psi_{i j}\left(z_{i}\right) \leq b_{i j}
\end{gathered}
$$

where

$$
\psi_{i j}\left(z_{i}\right)=\frac{\partial \varphi_{i}}{\partial x_{j}}\left(z_{i}\right), \mathscr{H}_{i j}=e_{q}(i) e_{n}^{T}(j) .
$$

Notice that this lemma is obvious from the mean value theorem, but it is important to introduce it at this stage, under 
TABLE I

MOdel PARAMETERS

\begin{tabular}{llll}
\hline \hline Acronyms & Definition & Units & Value \\
\hline$\alpha$ & Proportion of dilution rate for bacteria & $\mathrm{mmol} / \mathrm{l}$ & 0.5 \\
$k_{1}$ & Yield for substrate $\left(x_{1}\right)$ degradation & $\mathrm{g} /\left(\mathrm{g}\right.$ of $\left.x_{2}\right)$ & 42,1 \\
$k_{2}$ & Yield for VFA $\left(x_{3}\right)$ production & $\mathrm{mmol} /\left(\mathrm{g}\right.$ of $\left.x_{2}\right)$ & 116,5 \\
$k_{3}$ & Yield for VFA consumption & $\mathrm{mmol} /\left(\mathrm{g}\right.$ of $\left.x_{4}\right)$ & 268 \\
$k_{4}$ & Yield for $\mathrm{co}_{2}$ production & $\mathrm{mmol} / \mathrm{g}$ & 100 \\
$k_{5}$ & Yield for $\mathrm{co}$ production & $\mathrm{mmol} / \mathrm{g}$ & 300 \\
$k_{6}$ & Yield for $\mathrm{ch}$ production & $\mathrm{mmol} / \mathrm{g}$ & 302 \\
$\bar{\mu}_{1}$ & Maximum acidogenic bacteria $\left(x_{2}\right)$ growth rate & $1 / \mathrm{day}$ & 1,25 \\
$\bar{\mu}_{2}$ & Maximum methanogenic bacteria $\left(x_{4}\right)$ growth rate & $1 / \mathrm{day}$ & 0,74 \\
$k_{s_{1}}$ & Half saturation constant associated with $x_{1}$ & $\mathrm{~g} / \mathrm{l}$ & 0,41 \\
$k_{s 3}$ & Half saturation constant associated with $x_{3}$ & $\mathrm{mmol} / \mathrm{l}$ & 8,42 \\
$k_{i 3}$ & Inhibition constant associated with $x_{3}$ & $\mathrm{mmol} / \mathrm{l}$ & 247 \\
$k_{b}$ & Acidity constant of bicarbonate & $\mathrm{mol} / \mathrm{l}$ & $6,5.10^{-7}$ \\
$K_{H}$ & Henry's constant & $\mathrm{mmole} /(\mathrm{l} . \mathrm{atm})$ & 27 \\
$R$ & Gas constant & $\mathrm{L.atm} /(\mathrm{K} . \mathrm{mol})$ & 82,1 \\
$P_{T}$ & Total preasure & atm & 1,013 \\
$\mathrm{~T}$ & Temperature & Kelvin & 308 \\
$\gamma$ & Dimensionless parameter introduced by Hess $[11]$ & - & 0,025 \\
\hline \hline
\end{tabular}

this formulation, in the aim to simplify the presentation of the proposed observer design method. Indeed, for our technique, we will exploit (7)-(8) instead of a direct use of Lipschitz property.

Lemma 3.2 ([25]): Let $X$ and $Y$ two given matrices of appropriate dimensions. Then, for any symmetric positive definite matrix $S$ of appropriate dimension, the following inequality holds:

$$
X^{T} Y+Y^{T} X \leq \frac{1}{2}[X+S Y]^{T} S^{-1}[X+S Y] .
$$

This lemma will be very useful for the main contributions of this paper. It allows providing less restrictive LMI conditions compared to the classical LMI techniques for the considered class of systems.

\section{B. System description and assumptions}

Being motivated by the model of AD (1a-1f), we will investigate the general class of systems described by the following equations

$$
\left\{\begin{array}{l}
\dot{x}=A(\rho) x+B \gamma(x)+g(u, t) \\
y=C x
\end{array}\right.
$$

where $x \in \mathbb{R}^{n}$ is the state vector, $y \in \mathbb{R}^{p}$ is the output measurement, $u \in \mathbb{R}^{q}$ is an input vector and $\rho \in \mathbb{R}^{s}$ is an $\mathscr{L}_{\infty}$ bounded and known parameter. The affine matrix $A(\rho)$ is expressed under the form

$$
A(\rho)=A_{0}+\sum_{j=1}^{s} \rho_{j} A_{j}
$$

with $\rho_{j, \min } \leq \rho_{j} \leq \rho_{j, \max }$, which means that the parameter $\rho$ belongs to a bounded convex set for which the set of $2^{s}$ vertices can be defined by

$$
\mathbb{V}_{\rho}=\left\{\rho \in \mathbb{R}^{s}: \rho_{j} \in\left\{\rho_{j, \min }, \rho_{j, \max }\right\}\right\} .
$$

The matrices $A_{i} \in \mathbb{R}^{n \times n}, B \in \mathbb{R}^{n \times m}$ and $C \in \mathbb{R}^{p \times n}$ are constant. The non-linear function $\gamma: \mathbb{R}^{n} \longrightarrow \mathbb{R}^{m}$ is assumed to be globally Lipschitz. It is obvious that $B \gamma($.$) can always$ be written under the detailed form

$$
B \gamma(x)=\sum_{i=1}^{m} B_{i} \gamma_{i}(\overbrace{\mathscr{H}}^{\vartheta_{i} x})
$$

where $\mathscr{H}_{i} \in \mathbb{R}^{n_{i} \times n}$.

\section{Observer structure and error dynamics}

Now, we give the structure of the proposed invariant like nolinear state observer that will be used to estimate the unmeasurable state variables of the system (10).

Consider the following general observer structure

$$
\begin{aligned}
\dot{\hat{x}}= & A(\rho) \hat{x}+\sum_{i=1}^{m} B_{i} \gamma_{i}\left(\hat{\vartheta}_{i}\right)+g(y, u)+ \\
& L(\rho)\left[\ln \left(\frac{y_{1}}{e_{p}^{T}(1) C \hat{x}}\right), \ldots, \ln \left(\frac{y_{p}}{e_{p}^{T}(p) C \hat{x}}\right)\right]^{T}
\end{aligned}
$$

with

$$
\hat{\vartheta}_{i}=\mathscr{H}_{i} \hat{x}
$$

and

$$
L(\rho)=L_{0}+\sum_{j=1}^{s} \rho_{j} L_{j}
$$

where $\hat{x}$ is the estimate of $x$. The matrices $L_{i} \in \mathbb{R}^{n \times p}$ and the vector $e_{p}(i)=(\underbrace{0, \ldots, 0, \overbrace{1}^{i \text { th }}, 0, \ldots, 0}_{p \text { components }})^{T} \in \mathbb{R}^{p}, p \geq 1$. The observer parameters are to be determined so that the estimation error $e=x-\hat{x}$ converges asymptotically towards zero. Its dynamic is obtained by using equations (10) and (14)

$$
\begin{aligned}
\dot{e}= & A(\rho) e+\sum_{i=1}^{m} B_{i}\left(\gamma_{i}\left(\hat{\vartheta}_{i}\right)-\gamma_{i}\left(\vartheta_{i}\right)\right)- \\
& L(\rho)\left[\ln \left(\frac{y_{1}}{e_{p}^{T}(1) C \hat{x}}\right), \ldots, \ln \left(\frac{y_{p}}{e_{p}^{T}(p) C \hat{x}}\right)\right]^{T}
\end{aligned}
$$


At this stage, we will proceed step by step.

First, since $\gamma($.$) is globally Lipschitz, then from Lemma$ 3.1 there exist $r_{i} \in \operatorname{Co}\left(\vartheta_{i}, \hat{\vartheta}_{i}\right)$, functions $\phi_{i j}: \mathbb{R}^{n_{i}} \longrightarrow \mathbb{R}$ and constants $a_{i j}, b_{i j}$, such that

$$
B(\gamma(x)-\gamma(\hat{x}))=\sum_{i, j=1}^{m, n_{i}} \phi_{i j}\left(r_{i}\right) \mathscr{H}_{i j}\left(\vartheta_{i}-\hat{\vartheta}_{i}\right)
$$

and

$$
a_{i j} \leq \phi_{i j}\left(r_{i}\right) \leq b_{i j}
$$

where

$$
\phi_{i j}\left(r_{i}\right)=\frac{\partial \gamma_{i}}{\partial \vartheta_{i}^{j}}\left(r_{i}\right), \mathscr{H}_{i j}=B_{i} e_{n_{i}}^{T}(j)
$$

For shortness, we set $\phi_{i j} \triangleq \phi_{i j}\left(r_{i}\right)$. Without loss of generality, we assume that $a_{i j}=0$ for all $i=1, \ldots, m$ and $j=1, \ldots, n_{i}$ and moreover, since $\vartheta_{i}-\hat{\vartheta}_{i}=\mathscr{H}_{i} e$, then we have

$$
B(\gamma(x)-\gamma(\hat{x}))=\sum_{i, j=1}^{m, n_{i}} \phi_{i j} \mathscr{H}_{i j} \mathscr{H}_{i} e
$$

Second, we can always write

$$
\ln \left(\frac{y_{i}}{e_{p}^{T}(i) C \hat{x}}\right)=\underbrace{\ln \left(e_{p}^{T}(i) C x\right)-\ln \left(e_{p}^{T}(i) C \hat{x}\right)}_{\Upsilon_{i}(x)-\Upsilon_{i}(\hat{x})}
$$

thus, from Lemma 3.1 and in some specific invariant space there exist $z_{i} \in \operatorname{Co}\left(\min \left(x_{i}, \hat{x}_{i}\right), \max \left(x_{i}, \hat{x}_{i}\right)\right)$, functions $\psi_{i j}: \mathbb{R} \longrightarrow \mathbb{R}$ and constants $\min \left(\psi_{i j}\right), \max \left(\psi_{i j}\right)$, such that

$$
\Upsilon(x)-\Upsilon(\hat{x})=\sum_{i, j=1}^{p, n} \psi_{i j}\left(z_{i}\right) M_{i j} e
$$

with $\Upsilon(x)=\left[\Upsilon_{i}(x), \ldots, \Upsilon_{p}(x)\right]^{T}$ and

$$
\min \left(\psi_{i j}\right) \leq \psi_{i j}\left(z_{i}\right) \leq \max \left(\psi_{i j}\right)
$$

where

$$
\psi_{i j}\left(z_{i}\right)=\frac{\partial \Upsilon_{i}}{\partial x_{j}}\left(z_{i}\right), M_{i j}=e_{p}(i) e_{n}^{T}(j)
$$

For shortness, we set $\psi_{i j} \triangleq \psi_{i j}\left(z_{i}\right)$.

Now, using equations (17), (21) and (22), we obtain

$$
\dot{e}=\left(A(\rho)+\sum_{i, j=1}^{m, n_{i}} \phi_{i j} \mathscr{H}_{i j} \mathscr{H}_{i}-L(\rho) \sum_{i, j=1}^{p, n} \psi_{i j} M_{i j}\right) e
$$

Since, we know that $\min \left(\psi_{i j}\right) \neq 0$ in equation (23), we propose to write

$$
0 \leq \psi_{i j}-\min \left(\psi_{i j}\right) \leq \underbrace{\max \left(\psi_{i j}\right)-\min \left(\psi_{i j}\right)}_{\bar{b}_{i j}}
$$

and

$$
\begin{aligned}
\dot{e}= & \left(A(\rho)+\sum_{i, j=1}^{m, n_{i}} \phi_{i j} \mathscr{H}_{i j} \mathscr{H}_{i}-\right. \\
& \left.L(\rho) \sum_{i, j=1}^{p, n}\left(\psi_{i j}+\min \left(\psi_{i j}\right)-\min \left(\psi_{i j}\right)\right) M_{i j}\right) e
\end{aligned}
$$

hence

$$
\begin{aligned}
\dot{e}= & (A(\rho)-L(\rho) \underbrace{\sum_{i, j=1}^{p, n} \min \left(\psi_{i j}\right) M_{i j}+}_{\bar{C}} \\
& \sum_{i, j=1}^{m, n_{i}} \phi_{i j} \mathscr{H}_{i j} \mathscr{H}_{i}-L(\rho) \sum_{i, j=1}^{p, n} \underbrace{\left(\psi_{i j}-\min \left(\psi_{i j}\right)\right)}_{\varphi_{i j}} M_{i j}) e
\end{aligned}
$$

where

$$
0 \leq \varphi_{i j} \leq \bar{b}_{i j}
$$

The aim consists in finding the observer parameters so that the estimation error dynamics (28) be asymptotically stable.

Therefore, let us consider the classical quadratic Lyapunov function $V(e)=e^{T} \mathbb{P} e, \quad \mathbb{P}=\mathbb{P}^{T}>0$. By calculating its derivative along the trajectories of (28), we obtain

$$
\begin{aligned}
& \dot{V}=e^{T}\left(A(\rho)-L(\rho) \bar{C}+\sum_{i, j=1}^{m, n_{i}} \phi_{i j} \mathscr{H}_{i j} \mathscr{H}_{i}-L(\rho) \sum_{i, j=1}^{p, n} \varphi_{i j} M_{i j}\right)^{T} \mathbb{P} e \\
& +e^{T} \mathbb{P}\left(A(\rho)-L(\rho) \bar{C}+\sum_{i, j=1}^{m, n_{i}} \phi_{i j} \mathscr{H}_{i j} \mathscr{H}_{i}-L(\rho) \sum_{i, j=1}^{p, n} \varphi_{i j} M_{i j}\right) e \\
& \dot{V}=e^{T}(\underbrace{A^{T}(\rho) \mathbb{P}-\bar{C}^{T} L^{T}(\rho) \mathbb{P}+\mathbb{P} A(\rho)-\mathbb{P} L(\rho) \bar{C}}_{\Psi}) e \\
& +e^{T} \sum_{i, j=1}^{m, n_{i}} \phi_{i j}(\underbrace{\mathbb{P} \mathscr{H}_{i j}}_{\mathbb{X}_{i j}^{T}} \underbrace{\mathscr{H}_{i}}_{\mathbb{Y}_{i}}+\mathscr{H}_{i}^{T} \mathscr{H}_{i j}^{T} \mathbb{P}) e \\
& +e^{T} \sum_{i, j=1}^{p, n} \varphi_{i j}(\underbrace{\mathbb{P} L(\rho)}_{\overline{\mathbb{X}}_{i j}^{T}} \underbrace{\left(-M_{i j}\right)}_{\overline{\mathbb{Y}}_{i j}}+\left(-M_{i j}\right)^{T} L^{T}(\rho) \mathbb{P}) e
\end{aligned}
$$

Now, by applying Lemma 3.2 we have

$$
\mathbb{X}_{i j}^{T} \mathbb{Y}_{i}+\mathbb{Y}_{i}^{T} \mathbb{X}_{i j} \leq \frac{1}{2}\left(\mathbb{X}_{i j}+\mathbb{S}_{i j} \mathbb{Y}_{i}\right)^{T} \mathbb{S}_{i j}^{-1} \overbrace{\left(\mathbb{X}_{i j}+\mathbb{S}_{i j} \mathbb{Y}_{i}\right)}^{\Pi_{i j}}
$$

and

$$
\overline{\mathbb{X}}_{i j}^{T} \overline{\mathbb{Y}}_{i j}+\overline{\mathbb{Y}}_{i j}^{T} \overline{\mathbb{X}}_{i j} \leq \frac{1}{2}\left(\overline{\mathbb{X}}_{i j}+\overline{\mathbb{S}}_{i j} \overline{\mathbb{Y}}_{i j}\right)^{T} \overline{\mathbb{S}}_{i j}^{-1} \overbrace{\left(\overline{\mathbb{X}}_{i j}+\overline{\mathbb{S}}_{i j} \overline{\mathbb{Y}}_{i j}\right)}^{\bar{\Pi}_{i j}}
$$

for any symmetric positive definite matrices $\mathbb{S}_{i j}$ and $\overline{\mathbb{S}}_{i j}$. Moreover, from (19) and (29) and the fact that $a_{i j}=0$, inequality $\dot{V}<0$ holds if

$$
\begin{aligned}
\Psi- & \sum_{i, j=1}^{m, n_{i}}\left(\Pi_{i j}^{T}\left(-\frac{2}{b_{i j}} \mathbb{S}_{i j}\right)^{-1} \Pi_{i j}\right)- \\
& \sum_{i, j=1}^{p, n}\left(\bar{\Pi}_{i j}^{T}\left(-\frac{2}{\bar{b}_{i j}} \overline{\mathbb{S}}_{i j}\right)^{-1} \bar{\Pi}_{i j}\right)<0 .
\end{aligned}
$$


consequently, by Schur lemma, inequality (34) is equivalent to

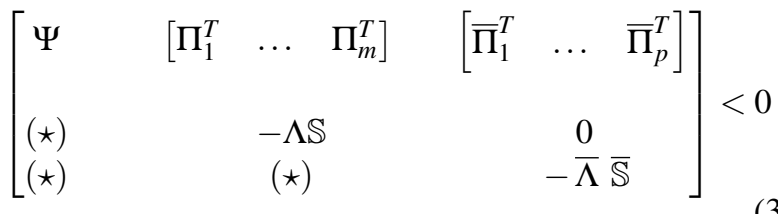

where

$$
\begin{gathered}
\Pi_{i}=\left[\Pi_{11}^{T} \ldots \Pi_{1 n_{i}}^{T}\right]^{T}, \quad \Pi_{i j}=\mathscr{H}_{i j}^{T} \mathbb{P}+\mathbb{S}_{i j} \mathscr{H}_{i} \\
\bar{\Pi}_{i}=\left[\bar{\Pi}_{11}^{T} \ldots \bar{\Pi}_{1 n}^{T}\right]^{T}, \quad \bar{\Pi}_{i j}=L^{T}(\rho) \mathbb{P}+\overline{\mathbb{S}}_{i j}\left(-M_{i j}\right)
\end{gathered}
$$

and

$$
\begin{gathered}
\mathbb{S}=\operatorname{block-diag}\left(\mathbb{S}_{1}, \ldots, \mathbb{S}_{m}\right), \quad \mathbb{S}_{i}=\operatorname{block-diag}\left(\mathbb{S}_{i 1}, \ldots, \mathbb{S}_{i n_{i}}\right) \\
\Lambda=\operatorname{block-diag}\left(\Lambda_{1}, \ldots, \Lambda_{m}\right), \quad \Lambda_{i}=\operatorname{block-diag}\left(\Lambda_{i 1}, \ldots, \Lambda_{i n_{i}}\right)
\end{gathered}
$$

with

$$
\Lambda_{i j}=\frac{2}{b_{i j}} \mathbb{I}_{n_{i}}
$$

and

$$
\begin{gathered}
\overline{\mathbb{S}}=\operatorname{block}-\operatorname{diag}\left(\overline{\mathbb{S}}_{1}, \ldots, \overline{\mathbb{S}}_{p}\right), \quad \overline{\mathbb{S}}_{i}=\operatorname{block}-\operatorname{diag}\left(\overline{\mathbb{S}}_{i 1}, \ldots, \overline{\mathbb{S}}_{i n}\right) \\
\bar{\Lambda}=\text { block-diag }\left(\bar{\Lambda}_{1}, \ldots, \bar{\Lambda}_{p}\right), \quad \bar{\Lambda}_{i}=\text { block-diag }\left(\bar{\Lambda}_{i 1}, \ldots, \bar{\Lambda}_{i n}\right)
\end{gathered}
$$

with

$$
\bar{\Lambda}_{i j}=\frac{2}{\bar{b}_{i j}} \mathbb{I}_{p}
$$

Finally, we use the change of variables $\mathscr{R}_{i}=L_{i}^{T} \mathbb{P}$ to solve the LMI (35).

\section{Simulation Results}

In order to apply the designed observer (14) to the AD model presented in Section II for estimating its key state variables, we first write the model in the form (10). This can be easily done by using the following parameters:

$$
\begin{aligned}
& \rho=u_{\text {out }}, \quad A_{0}=0, \quad A_{1}=-\operatorname{block}-\operatorname{diag}(1, \alpha, 1, \alpha, 1,1) \\
& B=\left[\begin{array}{cccccc}
-k_{1} & 1 & k_{2} & 0 & k_{4} & 0 \\
0 & 0 & -k_{3} & 1 & k_{5} & 0
\end{array}\right]^{T} \\
& \gamma(x)=\left[\begin{array}{l}
\mu_{1}\left(x_{1}\right) x_{2} \\
\mu_{2}\left(x_{3}\right) x_{4}
\end{array}\right] \\
& g(y, u)=\left[\begin{array}{c}
u_{1} S_{1 \text { in }} \\
0 \\
u_{1}\left(S_{2 i n}+S_{2 a d}\right) \\
0 \\
u_{1} C_{i n}-Q_{c} \\
u_{1} Z_{\text {in }}+u_{2} Z_{a d}
\end{array}\right]
\end{aligned}
$$

and for the selected outputs in (5):

$$
C=\left[\begin{array}{llllll}
1 & 0 & 0 & 0 & 0 & 0 \\
0 & 0 & 1 & 0 & 0 & 0 \\
0 & 0 & 0 & 0 & 0 & 1
\end{array}\right]
$$

Moreover, for the observer design we have, $m=2, s=1$, $n_{i}=2, \gamma_{1}(x)=\mu_{1}\left(x_{1}\right) x_{2}, \gamma_{2}(x)=\mu_{2}\left(x_{3}\right) x_{4}$,

$$
\begin{aligned}
& H_{1}=\left[\begin{array}{llllll}
1 & 0 & 0 & 0 & 0 & 0 \\
0 & 1 & 0 & 0 & 0 & 0
\end{array}\right] \\
& H_{2}=\left[\begin{array}{llllll}
0 & 0 & 1 & 0 & 0 & 0 \\
0 & 0 & 0 & 1 & 0 & 0
\end{array}\right] \\
& B_{1}=\left[\begin{array}{llllll}
-k_{1} & 1 & k_{2} & 0 & k_{4} & 0
\end{array}\right]^{T} \\
& B_{2}=\left[\begin{array}{llllll}
0 & 0 & -k_{3} & 1 & k_{5} & 0
\end{array}\right]^{T}
\end{aligned}
$$

The matrices $M_{i j} \in \mathbb{R}^{p \times n}$ with all elements null except the element $M_{i j}(i, j)$, for example:

$$
M_{23}=\left[\begin{array}{llllll}
0 & 0 & 0 & 0 & 0 & 0 \\
0 & 0 & 1 & 0 & 0 & 0 \\
0 & 0 & 0 & 0 & 0 & 0
\end{array}\right]
$$

The simulation have been run for $\rho_{\text {min }}=0.12$ day $^{-1}, \rho_{\text {max }}=$ $0.5 \mathrm{day}^{-1}, S_{1 \text { in }}=12.19 \mathrm{~g} / \mathrm{l}, S_{2 \text { in }}=6.7 \mathrm{mmol} / 1, C_{\text {in }}=58.08$ $\mathrm{mmol} / 1, Z_{\text {in }}=31 \mathrm{mmol} / 1, Z_{a d}=80 \mathrm{mmol} / 1, S_{2 a d}=11$ $\mathrm{mmol} / \mathrm{l}$, and the parameter values given in Table I. After solving the LMIs (35) by using LMI MATLAB Toolbox, the following observer gains have been obtained

$$
\begin{aligned}
L_{0}= & {\left[\begin{array}{ccc}
10.9834 & -3.2478 & -0.0073 \\
-0.2685 & 0.0822 & 0.0043 \\
-29.9616 & 272.6428 & -6.0918 \\
-0.0014 & -1.0061 & 0.0276 \\
-26.5279 & -287.3792 & 6.8599 \\
-0.0001 & 0.0001 & 0.0019
\end{array}\right] } \\
L_{1}= & {\left[\begin{array}{ccc}
6.3587 & 0.5083 & -0.1598 \\
-0.1565 & -0.0123 & 0.0038 \\
-15.8649 & -0.4543 & -1.7419 \\
-0.0070 & -0.0036 & 0.0082 \\
-17.6868 & -2.3044 & 2.8363 \\
0.0000 & 0.0001 & -0.0039
\end{array}\right] }
\end{aligned}
$$

Moreover, the control input $u_{1}$ has been varied during the simulation as represented in Figure 1. The system and the observer were initialized, respectively, by $x(0)=$ $[1.8,0.15,3,0.8,65,62]^{T}$ and $\hat{x}(0)=[1.8,0.2,3,1,60,62]^{T}$. Simulation results are depicted in Figures 2-4. It is quite clear from the later figures that the estimated state variables by the proposed observer converge to the simulated system states. This shows the effectiveness of the proposed methodology.

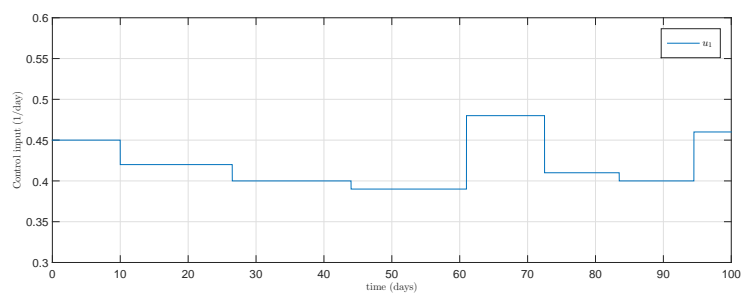

Fig. 1. Control input $u_{1}(1 /$ day $)$. 


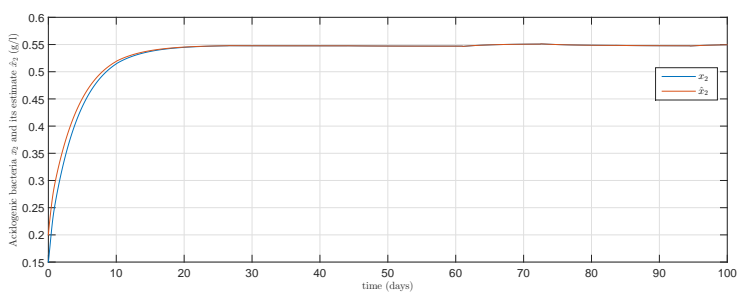

Fig. 2. Acidogenic bacteria $x_{2}$ and its estimate $\hat{x}_{2}(g / l)$.

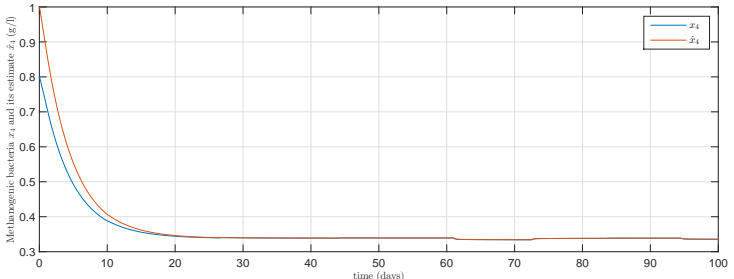

Fig. 3. Mathenogenic bacteria $x_{4}$ and its estimate $\hat{x}_{4}(g / l)$.

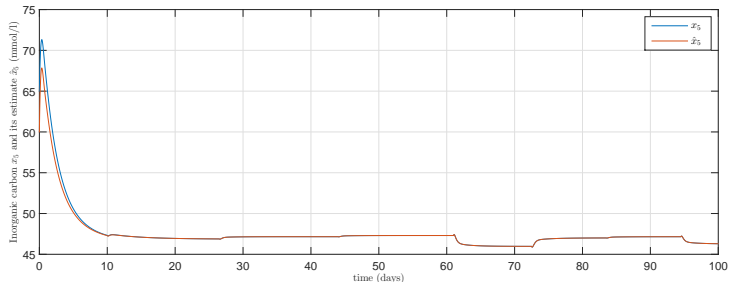

Fig. 4. Inorganic carbon $x_{5}$ and its estimate $\hat{x}_{5}(\mathrm{mmol} / \mathrm{l})$.

\section{CONCLUSION}

In this note, we have designed a novel invariant like nonlinear state observer for a two step AD model. In order to guarantee the asymptotic convergence of the estimation error to zero, we have used an LMI approach. In the synthesized LMI conditions, we have included additional decision variables to enhance its feasibility. This was possible due to the use of a suitable reformulation of the Young's inequality. To illustrate the effectiveness of the methodology, we have run numerical simulation which has shown satisfactory results. This opens new prospects to evaluate performances of the proposed observer in presence of disturbances in the system and noise in measurements.

\section{REFERENCES}

[1] N. Aghannan and P. Rouchon. On Invariant Asymptotic Observers. In In Proceedings of the 41st IEEE Conference on Decision and Control, volume 2, pages 1479-1484, 2002.

[2] R. Antonelli and A. Astolfi. Nonlinear controllers design for robust stabilization of continuous biological reactors. In Control Applications, 2000. Proceedings of the 2000 IEEE International Conference on, pages 760-765. IEEE, 2000.

[3] G. Bastin and D. Dochain. On-line Estimation and Adaptive Control of Bioreactors. Process Measurement and Control. Elsevier, 1990.

[4] O. Bernard and J.L Gouzé. Closed loop observers bundle for uncertain biotechnological models. Journal of Process Control, 14(7):765-774, 2004

[5] Ibtissem Didi, Hacen Dib, and Brahim Cherki. An invariant observer for a chemostat model. Automatica, 50(9):2321-2326, 2014.
[6] D. Dochain. State and parameter estimation in chemical and biochemical processes: a tutorial. Journal of Process Control, 13(8):801 - 818, 2003.

[7] K.C. Draa, H. Voos, M. Alma, and M. Darouach. Invariant observer applied to anaerobic digestion model. In 2016 IEEE 21st International Conference on Emerging Technologies and Factory Automation (ETFA), pages 1-4, 2016.

[8] L. Dewasme J.A. Moreno JA E. Rocha-Cozatl, M. Sbarciog and A. Vande Wouwer. State and input estimation of an anaerobic digestion reactor using a continuous-discrete unknown input observer. IFACPapersOnLine, 48(8):129-134, 2015.

[9] R. Bakke F. Haugen and B. Lie. State estimation and model-based control of a pilot anaerobic digestion reactor. Journal of Control Science and Engineering, 2014:3, 2014.

[10] M.Z. Hadj-Sadok and J.L. Gouzé. Estimation of uncertain models of activated sludge processes with interval observers. Journal of Process Control, 11(3):299-310, 2001

[11] J. Hess. Modélisation de la Qualité du Biogas Produit par un Fermenteur Méthanogène et sa Stratégie de Régulation en Vue de sa Valorisation. PhD thesis, Université de Nice - Sophia Antipolis, 2008

[12] F. Miens J. Harmand and J.P. Steyer. High gain observer for diagnosing an anaerobic fixed bed reactor. In Control Conference (ECC), 2001 European, pages 2829-2834. IEEE, 2001.

[13] M.A. Hussain J. Mohd Ali, N.H. Hoang and D. Dochain. Review and classification of recent observers applied in chemical process systems. Computers \& Chemical Engineering, 76:27 - 41, 2015.

[14] M. Darouach K. Chaib Draa, H. Voos and M.Alma. A formal modeling framework for anaerobic digestion systems. In Proceedings of the $201517^{\text {th }}$ UKSIM-AMSS International Conference on Modelling and Simulation, pages 426 - 431. IEEE Computer Society, 2015.

[15] J. Ma L. Yu, P.C Wensel and S. Chen. Mathematical modeling in anaerobic digestion (ad). Journal of Bioremediation \& Biodegradation, 2014, 2013

[16] K.J. Keesman L.J.S. Lukasse and G. van Straten. A recursively identified model for short-term predictions of nh4/no3 concentrations in alternating activated sludge processes. Journal of Process Control, 9(1):87 - 100, 1999.

[17] M. Lombardi, K. Fiaty, and P. Laurent. Implementation of observer for on-line estimation of concentration in continuous-stirred membrane bioreactor: Application to the fermentation of lactose. Chemical Engineering Science, 54(13):2689 - 2696, 1999.

[18] R. Moletta. Technologies de traitement des effluents industriels par la méthanisation. La méthanisation (2e ed.), page 133, 2011.

[19] B. Chachuat O. Bernard and J.Ph. Steyer. State estimation for wastewater treatment processes. Wiley, Oxford, UK, 2007.

[20] D. Dochain A. Genovesi O. Bernard, Z. Hadj-Sadok and J.Ph. Steyer. Dynamical model development and parameter identification for an anaerobic wastewater treatment process. Biotechnology and bioengineering, 75(4):424-438, 2001

[21] S.L. Shah R.D. Gudi and M.R. Gray. Adaptive multirate state and parameter estimation strategies with application to a bioreactor. AIChE Journal, 41(11):2451-2464, 1995.

[22] P. Martin S. Bonnabel and P. Rouchon. Symmetry-preserving observers. ArXiv Mathematics e-prints, 2006. Provided by the SAO/NASA Astrophysics Data System.

[23] P. Weiland. Biogas production: current state and perspectives. Applied microbiology and biotechnology, 85(4):849-860, 2010.

[24] A. Zemouche, M. Boutayeb, and G. I. Bara. Observers for a class of Lipschitz systems with extension to $\mathscr{H}_{\infty}$ performance analysis. Systems \& Control Letters, 57(1):18-27, 2008

[25] A. Zemouche, R. Rajamani, B. Boulkroune, H. Rafaralahy, and M. Zasadzinski. $\mathscr{H}_{\infty}$ circle criterion observer design for Lipschitz nonlinear systems with enhanced LMI conditions. In IEEE American Control Conference, Boston, MA, USA., July 2016. 